\title{
Estructura espacial de la movilidad residencial en la Región Metropolitana de Santiago de Chile. 2012-2017
}

\author{
Spatial structure of residential mobility in the \\ Metropolitan Region of Santiago de (Chile). 2012-2017
}

\author{
Severino Escolano Utrilla² (i), Jorge Ortiz Véliz³ (1) \\ y Rodrigo Moreno Mora ${ }^{4}$ (c)
}

\begin{abstract}
RESUMEN
La mayor parte de la investigación empírica sobre los patrones espaciales de movilidad residencial intraurbana se ha llevado a cabo a partir del estudio de los balances de entradas y salidas registradas en diversas unidades espaciales. Esta aproximación no permite delimitar áreas migratorias definidas según el origen, destino e intensidad de la movilidad residencial. Aquí se estudian los tipos espaciales de la movilidad residencial y su estructura en las comunas de la Región Metropolitana de Santiago (RMS) utilizando datos individuales del cambio de residencia entre comunas. La aplicación combinada de métodos de análisis espacial y de análisis de redes geosociales, ha puesto de manifiesto: a) el carácter complejo y bastante integrado de la red de movimientos residenciales; b) la gran influencia de la proximidad geográfica en los cambios de residencia y c) los efectos de los cambios de residencia en la diversificación socioespacial presentan notables diferencias territoriales. En conjunto, la estructura funcional y espacial de la movilidad residencial es coherente con los procesos de expansión y fragmentación física, social y funcional de la RMS.
\end{abstract}

Palabras clave: movilidad residencial intraurbana, redes socioespaciales, áreas de movilidad, región metropolitana, Santiago (Chile).

\begin{abstract}
Most empirical research on spatial patterns of intra-urban residential mobility has been based on the study of the inflow and outflow recorded in various spatial units. This approach does not allow for the definition of migratory areas by the origin, destination and intensity of residential mobility. Here the spatial types and structure of residential mobility in the communes of the Santiago Metropolitan Region (RMS) are studied using individual data on changes of residence between communes. Combining the application of spatial analysis methods and the analysis of geosocial networks has demonstrated: a) the complex and fairly well-integrated nature of the network of residential movements; b) the major influence of geographical proximity on changes of residence; and c) notable territorial differences in the effects of changes of residence on socio-spatial
\end{abstract}

Este artículo se ha realizado con el apoyo del proyecto de investigación nº CSO2016-74888-C4-3-R), del Programa Estatal de Investigación, Desarrollo e Innovación Orientada a los Retos de la Sociedad del Ministerio de Economía, Industria y Competitividad del Gobierno de España, y del Gobierno de Aragón con la financiación del Fondo Social Europeo.

Departamento de Geografía y Ordenación del Territorio, Universidad de Zaragoza (España): Correo electrónico: severino@unizar.es Departamento de Geografía. Facultad de Arquitectura y Urbanismo. Universidad de Chile: Correo electrónico: jortiz@uchilefau.cl Departamento de Geografía. Facultad de Arquitectura y Urbanismo. Universidad de Chile: Correo electrónico: romoreno@uchilefau.cl 
diversification. As a whole, the functional and spatial structure of residential mobility is consistent with the processes of expansion and physical, social and functional fragmentation of the RMS.

Keywords: Intraurban residential mobility; geospatial networks, neighbourhood, metropolitan regions, Santiago (Chile).

Los científicos sociales han estudiado los procesos de la movilidad de la población, a diversas escalas, para conocer las características de quién cambia de residencia (o permanece en la misma), por qué razones lo hace, cómo se producen estos cambios y, en bastante menor medida, entre qué lugares se producen (Di Virgilio 2011; King, 2012), aunque la investigación de la movilidad residencial intraurbana ha recibido menos atención que otras movilidades, como, por ejemplo, la internacional (Coulter el al., 2015).

El estudio de la movilidad residencial intraurbana se ha centrado en el análisis de las decisiones individuales y familiares de cambio y elección de nueva residencia, tomadas en el marco de la maximización de la satisfacción de las aspiraciones residenciales. Este punto de vista considera la movilidad residencial como un proceso de ajuste espacial. Abundantes estudios empíricos llevados a cabo con este enfoque han documentado el tipo e intensidad de la influencia de diferentes factores que impulsan o limitan la movilidad residencial intraurbana. Entre otros, los cambios de residencia se han relacionado con la dinámica y políticas de los mercados de la vivienda (Andrews \& Caldera, 2011; Van der Vlist, Czamanski, Folmer, 2011), las etapas del ciclo de vida Clark \& Dieleman, 1996; Pujadas et al., 2007), el estatus socioeconómico de los sujetos que cambian de residencia, la distancia o accesibilidad al trabajo, las características del entorno (Bible \& Brown 1981; Brown \& Moore 1970; Brummell, 1979), la mejora de la accesibilidad al trabajo o a los servicios y equipamientos públicos (Morris et al., 2018), las oportunidades de inversión o cambios en el estilo de vida (Méndez \& Gayo 2018), o con razones emocionales (Raulin 2014; Villani 2014) y otros.

Los cambios en los estilos de vida y en la composición de los hogares, junto con diversificación epistemológica habida en las ciencias sociales desde la década de 1990 -giros en la Geografía: cultural, de la movilidad, estudios feministas-, han enriquecido el marco teórico y los datos empíricos de los estudios de la movilidad residencial (King, 2012), al tiempo que han impulsado el desplazamiento del enfoque desde el estudio de las decisiones individuales hasta la conceptualización de la movilidad residencial como un fenómeno social complejo (Torrado 2019, p. 17).

Desde las nuevas perspectivas, la movilidad residencial intraurbana se entiende como una de las prácticas materiales y simbólicas que más profundamente contribuyen a transformar la geografía social, funcional y urbanística de las ciudades, por cuanto la residencia es el centro de gravedad de los territorios cotidianos de los individuos y una referente central de la construcción de las identidades individuales y socioespaciales. La movilidad residencial se concibe como una práctica relacional que conecta vidas a través del tiempo y el espacio (Coulter el al., 2015) y que se desarrolla en un contexto en el que interactúan las preferencias y posibilidades individuales con las condiciones estructurales. En los nuevos enfoques, el territorio se puede considerar como resultado y parte constitutiva del proceso de movilidad residencial intraurbana, y no solo como una consecuencia necesaria del cambio de localización de la residencia o como una variable independiente de modelos de decisión, ya que los territorios de las prácticas cotidianas de las personas - el barrio, la vecindad-conforman, en conjunto, un elemento en las decisiones de 
cambio de residencia. La elección de una nueva vivienda en el mismo barrio o área que la vivienda actual supone ventajas como mantener las relaciones sociales, el sentimiento de pertenencia a una comunidad o minimizar el aprendizaje de nuevos espacios urbanos (Sun \& Manson, 2012).

En esta línea, el presente trabajo aborda el estudio de la estructura espacial de la movilidad residencial en la Región Metropolitana de Santiago de Chile (RMS), entendida como la organización funcional y espacial de un conjunto relacional de lugares, formado por las comunas de origen y destino de los cambios de residencia, que conectan en el espacio las trayectorias individuales de la movilidad residencial. Esta acepción implica que la distancia, dirección y proximidad entre la residencia actual y la nueva se relacionan con las características y formas de los cambios de residencia, en el sentido de que las migraciones espacialmente cercanas entre sí tienen alta probabilidad de presentar propiedades similares (autocorrelación espacial). Por otro lado, los migrantes con estándares sociales parecidos (estatus económico, nivel educativo, modelo familiar, etc.) es probable que muestren patrones migratorios semejantes, independientemente de donde residan (homophily).

La investigación tiene por objeto identificar y caracterizar los tipos de movimientos residenciales y su organización espacial en la RMS en el quinquenio 20122017, enmarcados en los procesos de expansión y reconfiguración recientes de la RMS. En la RMS, la movilidad residencial se ha relacionado con el grado de segregación residencial (Rodríguez Vignoli \& Rowe 2017), con cambios en la estructura demográfica (Rodríguez Vignoli \& Rowe 2018) y con los modos de vida de quienes cambian (o permanecen) de residencia (Álvarez, 2010). En menor medida se han estudiado las trayectorias y patrones espaciales de la movilidad residencial (Ortiz, Escolano 2013; Méndez, Gayo 2019) y no se han publicado investigaciones sobre la organización espacial de los cambios residenciales en la RMS considerados en conjunto. Con este contexto, cabe preguntarse por la relación de la organización espacial de la movilidad residencial con los procesos simultáneos de expansión y fragmentación física, social y funcional detectados en la periferia y centro del área metropolitana de Santiago (Borsdorf 2003; De Mattos et al., 2004; De Mattos \& Hidalgo, 2007; Borsdorf \& Hidalgo, 2010). De forma específica, se plantean los siguientes objetivos:

1. Caracterizar los tipos espaciales de movimientos residenciales en la RMS en el período 20122017, según su zona de origen y destino, orientación y distancia.

2. Identificar y caracterizar la configuración espacial de los patrones de movilidad residencial que emergen de las conexiones entre las comunas de origen y destino del cambio de residencia en la RMS en el período 20122017.

Esta investigación pretende contribuir al conocimiento de la movilidad residencial en tres aspectos. Primero, se presenta una tipología de movimientos residenciales, adaptada a la estructura de las grandes áreas metropolitanas latinoamericanas. Segundo, se ha elaborado un modelo de la movilidad residencial entre todas las comunas de la RMS con la distribución de las distancias de los cambios de residencia según la magnitud de estos, contrastada con la distribución de todas las distancias posibles ( $\mathrm{HO})$. Tercero, se delimitan las configuraciones geográficas que surgen de la intensidad de intercambios bidireccionales de residentes entre comunas. Por otro lado, el trabajo aporta una novedad metodológica, como es la aplicación de la metodología de análisis de 
redes geoespaciales para identificar y caracterizar la organización funcional y espacial de la red de movimientos residenciales.

La delimitación de áreas en función de la movilidad residencial es una aportación útil en sí misma, pues supone la aplicación de criterios dinámicos para explorar la estructura socioespacial de las ciudades frente al uso tradicional de atributos estáticos (Sun \& Manson, 2012). Esta información también es relevante para la formulación de políticas públicas en las principales áreas de la planificación y gobernanza urbanas.

El resto del artículo está organizado del modo siguiente. En la primera sección, se describe la metodología aplicada y los datos utilizados. En las dos siguientes, se presentan los resultados obtenidos. Después, se discuten y explican los resultados. Finalmente, se exponen las conclusiones y se proponen líneas de investigación futuras.

\section{Datos y métodos}

Los datos utilizados en esta investigación expresan el número de jefes de hogar que han cambiado de comuna de residencia entre 2012 y 2017 (y entre 1997 y 2002), en las 52 comunas de la RMS. La información se ha obtenido de las respuestas dadas a la pregunta de los censos: ¿En qué comuna o país vivía en abril de 2012 (o 1997)?, hecha a todas las personas de cinco años o más (INE 2017). Los datos se han extraído del servicio de microdatos del Instituto Nacional de Estadísticas (INE) mediante la aplicación RedAtam WebServer (INE). La categoría censal jefes de hogar se ha utilizado como unidad temática de análisis ya que el cambio de residencia está muy asociado a las familias.

De cada jefe de hogar se ha retenido su ocupación en una de las dos ramas de actividad siguientes: M. Actividades profesionales, científica y técnicas (profesionales) o T. Actividades de los hogares como empleadores; actividades no diferenciadas como productores de bienes y servicios para uso propio (no diferenciados). Se han tomado estas categorías como representativas, a priori, de diferentes patrones espaciales de movilidad. El total de jefes de hogar ocupados en la rama $M$ es de 84.776 (3,78\% del total de jefes de hogar de la RMS) y el de la rama $T 57.665$ (2,58\% del total de jefes de hogar de la RMS). Como estimador de estatus económico se ha utilizado el indicador «ingreso total del hogar» de la encuesta CASEN 2015 (Ministerio de Desarrollo Social y Familia). Los valores se han clasificado en 4 niveles, siguiendo la estructura de su distribución.

Con los datos individuales de la movilidad residencial de los jefes de hogar, agregados por comunas, se ha formado una matriz origendestino, que recoge los cambios de residencia entre cada una de las 52 comunas de RMS. A partir de esta matriz se ha elaborado una red geoespacial en la que los nodos representan a las comunas (los centroides de las comunas) y las aristas (conexiones) simbolizan la dirección origendestino y la magnitud de los cambios residenciales entre los nodos. Los nodos contienen información espacial y temática. Esta estructura de datos constituye el modelo de la movilidad residencial de la RMS.

La secuencia lógica de trabajo comprende dos fases: a) análisis y caracterización de la movilidad residencial según los tipos de movilidad, el nivel socioeconómico de las comunas de origen 
y destino del cambio de residencia y la distancia recorrida, y b) identificación de grupos funcionales de comunas (clústeres) y sus correspondientes áreas espaciales según la intensidad de sus intercambios residenciales.

A escala metropolitana, Torrado (2019, p. 18) distingue tres formas principales de movilidad residencial, cada una con implicaciones propias en los procesos de reconfiguración metropolitana: los movimientos de suburbanización, que se producen entre el centro y la franja externa de crecimiento metropolitano, los de centralización, desde la corona exterior hacia la ciudad central, y la movilidad entre los municipios de la corona. El municipio es considerado como la unidad espacial adecuada para el estudio de la movilidad residencial metropolitana.

A las tres formas de movilidad residencial anteriores se han añadido otras 5, acordes con la estructura socioespacial de la RMS. En primer lugar, se han delimitado los ámbitos espaciales siguientes: Centro, constituido por la comuna de Santiago; pericentro, formado por 9 comunas contiguas a la de Santiago; pericentro distante, que engloba 10 comunas adyacentes a las del pericentro; periferia compacta, que comprende 8 comunas contiguas al pericentro hasta el límite del tejido urbano continuo; periferia distante, formada por 18 comunas de la corona más externa de la RMS y sector noreste, compuesto por 6 comunas. Entre estas áreas se han identificado los siguientes tipos de movilidad: a) movimientos de centralización: los que se dirigen al centro o pericentro desde las demás áreas (excepto del noreste); b) movimientos de recentralización: tienen su origen y destino en el centro y los pericentros; c) movimientos de suburbanización: son los que tienen como destino las periferias y su origen en las demás áreas, excepto la noreste; d) movimientos laterales suburbanos: son los que se producen dentro y entre las periferias; e) movimientos del sector noreste: se han distinguido los que se dirigen desde este sector a las restantes áreas, los que llegan a este sector desde las demás áreas y los que tienen lugar dentro de este mismo sector. Adicionalmente, se han calculado y representado los movimientos con origen y destino en Santiago.

Con la información del nivel medio de ingresos de las comunas asociada a las trayectorias de los cambios de residencia se ha construido una tipología, inspirada en la propuesta de Bäing \& Wong (2012), que muestra la difusiónconcentración espacial del «capital social». Se consideran movimientos de "elitización» (gentrifiers) a los cambios de residencia desde las comunas de mayor ingreso a las de menor ingreso, y movimientos de «ascenso social» (escalators) a los producidos desde comunas de menor a mayor nivel de ingresos.

La distribución de las distancias de los cambios de residencia se ha obtenido a partir de la distancias ponderada de cada flujo residencial entre comunas, que se ha calculado con la siguiente ecuación:

$$
D_{i}=\sum_{i j}^{n} s_{i j} d_{i j} / \sum_{i j}^{n} s_{i j}
$$

Donde: $\mathrm{D}_{i}$ es la distancia media ponderada desde cada centroide de comuna al resto de los centroides; $\mathrm{s}_{i j}$ representa el volumen de residentes de la comuna $i$ que han cambiado su residencia a la comuna $j$; $d_{i j}$ : distancia geodésica entre las comunas $i$ y $j$.

De modo análogo, se ha calculado la distribución de todas las distancias posibles $(\mathrm{HO})$ de los movimientos de cambio de residencia. 
Para identificar y medir la estructura funcional y su patrón espacial se han utilizado solamente dos indicadores seleccionados, entre los múltiples descriptores de las redes geoespaciales, por su utilidad para los objetivos de este trabajo.

El primero es la centralidad eigenvector, calculada para cada nodo (centroide de cada comuna), cuya generalización es un estimador del valor de cada nodo como distribuidor (hub) de la red de movimientos residenciales. Un nodo con valor de centralidadhub elevado indica que sus emigrantes (conexiones hacia fuera) se dirigen a nodos que tienen valores altos de inmigración (conexiones hacia dentro). La centralidad eigenvector se calcula del modo siguiente:

Centralidad eigenvector: vector combinado de todos los valores escalados $\mathrm{V}_{\mathrm{k}}$ de una red.

Sean: $A$ una red unimodal de $\mathrm{N}$ nodos; $\mathrm{K}$ el número de componentes de la red $\mathrm{A} ; \mathrm{N}_{\mathrm{k}}$ nodos del componente kth; $V_{k}$ eigenvector dominante de la subred inducida por los nodos $N_{k}$. Se escalan los valores $V_{k}$ multiplicándolos por $\mathrm{N}_{\mathrm{k}} / \mathrm{N}$ (Bonacich 1972).

Centralidad hub = centralidad eigenvector de la red $A^{*} A ;($ Min =0; Max. =1) Generalización de la centralidad eigenvector (Kleinberg, 1998).

El segundo es la composición modular de la red, que es una propiedad fundamental de la estructura y funcionamiento de las redes. Entre los diversos métodos de detección de módulos (agrupamientos; clústeres) en redes complejas (Malliaros \& Vazirgiannis 2013), se ha utilizado el algoritmo de agrupamiento jerárquico de nodos basado en la similitud de la estructura de sus flujos (Clauset et al., 2004).

La modularidad es una magnitud que mide la fuerza con que una red está estructurada en módulos (Newman, 2006). Su cálculo relaciona, de forma normalizada, la fracción de conexiones entre los nodos de un grupo respecto de las que cabría esperar en una distribución aleatoria con el mismo número de elementos. Los valores de este indicador varían entre -0,5 y 1; el valor es positivo si las conexiones observadas son más que las esperadas por azar. Su ecuación es la siguiente:

$$
Q=\frac{1}{4 m} \sum_{i i}\left(A_{i j} \frac{k_{i} k_{j}}{2 m}\right) s_{i} s_{j}
$$

Donde: $s_{i}=1$ si el vértice $i$ pertenece al grupo 1 y $s_{i}=-1$ si pertenece al grupo $2 ; A_{i j}$ : número de aristas (conexiones) entre los vértices (nodos $i, j$ ); $A_{i j}$ elementos de la matriz de adyacencia; $k_{i} k$ $j / 2 m$ : número de aristas esperado entre los vértices $i$ y $j$ si aquellas se distribuyen aleatoriamente; $k_{i}$ y $k_{j}$ : grado de los vértices; $m=1 / 2 \sum_{i} k_{i}$ : número total de aristas de la red.

El índice E-I es un indicador normalizado de la cohesión de cada módulo que expresa la proporción de relaciones migratorias bilaterales entre las comunas del mismo grupo (conexiones internas: I) y las relaciones con las comunas que no forman parte del mismo grupo (conexiones externas: E). Su ecuación se puede generalizar para utilizar los pesos (migrantes: $w$ ) de las conexiones:

$$
E-I=\left(E_{w}-I_{w}\right) /\left(E_{w}+I_{w}\right)
$$


Su valor varía entre -1 , todas las conexiones son internas, (es un grupo aislado) hasta 1, todas las aristas son externas; el valor 0 , indica el mismo número de aristas internas y externas (o cambios residenciles).

Los indicadores de las georedes se han calculado con el programa ORANetScenes 3.7 (Carley, 2017) y el gráfico de la red se han elaborado con el programas Cytoscape 3.7 (Shannon et al. 2003). El análisis espacial y la cartografía se han realizado con el programa ArcGIS 10.5 (ESRI).

Los datos y métodos utilizados condicionan el alcance de los resultados. La pregunta censal tiene limitaciones bien conocidas: no estima los retornos y subestima los posibles cambios residenciales intercensales, pero es la fuente más completa y consistente para comparar datos temporales y espaciales sobre migraciones. No obstante, estas carencias no modifican los rasgos estructurales de la movilidad residencial (Rodríguez Vignoli, Rowe 2017, p. 25). Además, las distancias de los cambios de residencia están referidas a los centroides de cada comuna, por lo que son un promedio aproximado a la realidad, por la compensación de distancias cortas y largas.

\section{Diversidad de tipos espaciales de movilidad residencial y proximidad geográfica}

Entre 2012 y 2017, cambiaron de comuna de residencia dentro de la RMS 926.633 personas, de las cuales 318.870 eran jefes o jefas de hogar, es decir, un 14,2\% de los jefes de hogar residentes en la RMS en 2017. Como se aprecia en el cuadro No 1, la intensidad migratoria en la RMS es ligeramente inferior a la del promedio del país y decrece respecto del período 19972002, lo que corrobora la estabilidad del asentamiento de la población (Herrera-Ponce 2007).

Cuadro No 1

Algunas magnitudes de las migraciones intercomunales en Chile y la RMS. 2017

\begin{tabular}{|l|r|r|r|}
\hline & \multicolumn{1}{|c|}{ Chile } & \multicolumn{1}{|c|}{$\begin{array}{c}\text { R. Metropolitana de } \\
\text { Santiago }\end{array}$} & RMS/Chile (\%) \\
\hline Población & 17.574 .003 & 7.028 .680 & 40,0 \\
\hline Jefes o jefas de hogar & 5.651 .637 & 2.238 .179 & 39,6 \\
\hline Tasa de movilidad intercomunal (Tmi) $\left(^{*}\right)$ & & & \\
$2012-2017$ & 31,53 & 30,1 & \\
19972002 & 32,03 & 32,8 & \\
\hline Número de comunas & 346 & 52 & 15,0 \\
\hline
\end{tabular}

Fuente: INE: Censo de población y vivienda 2017. RedAtam Webserver.

(*) Tmi: $=($ Migrantes/5) $/($ población $2017+$ población 2012) / 2)* 1000

CELADE, Proyecto MIALC. Procesado con REDATAM7. 09-2018. Elaboración propia.

El volumen de los flujos de los movimientos residenciales de entrada y salida de las comunas muestra una asociación muy elevada con el total de jefes de hogar residentes en cada comuna $\left(R^{2}=0,9\right.$ en ambos casos). Ahora bien, mientras que las entradas de residentes presentan una relación lineal $\left(y=0,1026 x^{1,02}\right)$, la tendencia de las salidas es supralineal, es decir, de las comunas 
más grandes salen proporcionalmente más movimientos residenciales $\left(\mathrm{y}=0,0089 \mathrm{x}^{1,25}\right)$ (figura $\left.\mathrm{N}^{0} 1\right)$. Este modelo y sus parámetros son idénticos a los de la movilidad residencial total en relación con la población total.

Figura № 1.

Relación entre la movilidad residencial y el número de jefes de hogar de las comunas en la RMS. 2017

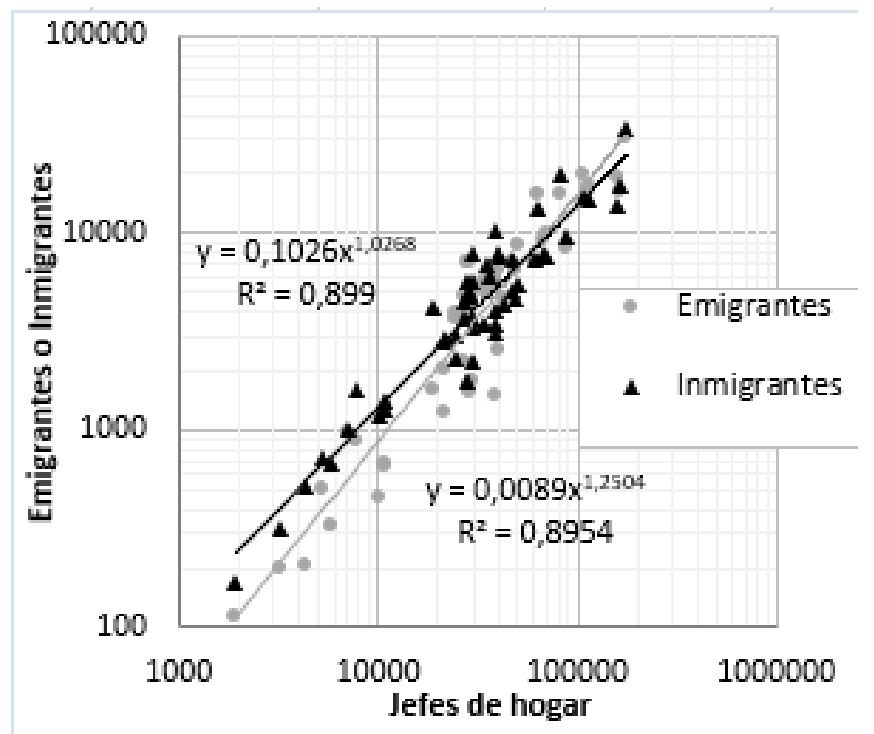

Fuente: INE: Censo de población y vivienda 2017. RedAtam Webserver. Elaboración propia.

El mapa de la figura No 2 muestra que el grueso de la movilidad residencial se despliega en las comunas del centro y sector este de la RMS, en el eje MaipúSantiagoLas Condes, que también son las comunas más pobladas. La principal concentración de flujos se localiza en las comunas del sector noreste (Providencia, Las Condes, Ñuñoa, Vitacura) con intensos intercambios mutuos de residentes. Otros agrupamientos están compuestos por dos o tres comunas con fuertes conexiones residenciales recíprocas (La FloridaPuente AltoSantiago; MaipúSantiago).

Sin embargo, los balances netos de movilidad presentan un patrón espacial de zonas concéntricas. En 2017, las comunas del centro (Santiago y otras contiguas del pericentro -San Miguel, Independencia y las restantes), diferentes en su composición social, tienen saldos positivos y se hallan rodeadas por un anillo de comunas con saldos de movilidad residencial negativos, incluidas las del sector noreste. Las comunas más periféricas tienen saldos positivos, en algunos casos con valores absolutos elevados. Esta distribución de los balances está producida por combinaciones complejas de flujos residenciales de entrada y salida, cuya magnitud y densidad varían espacialmente. 
Figura No 2

Movilidad residencial intercomunal de los jefes de hogar en la RMS: entradas, salidas y balance. 20122017
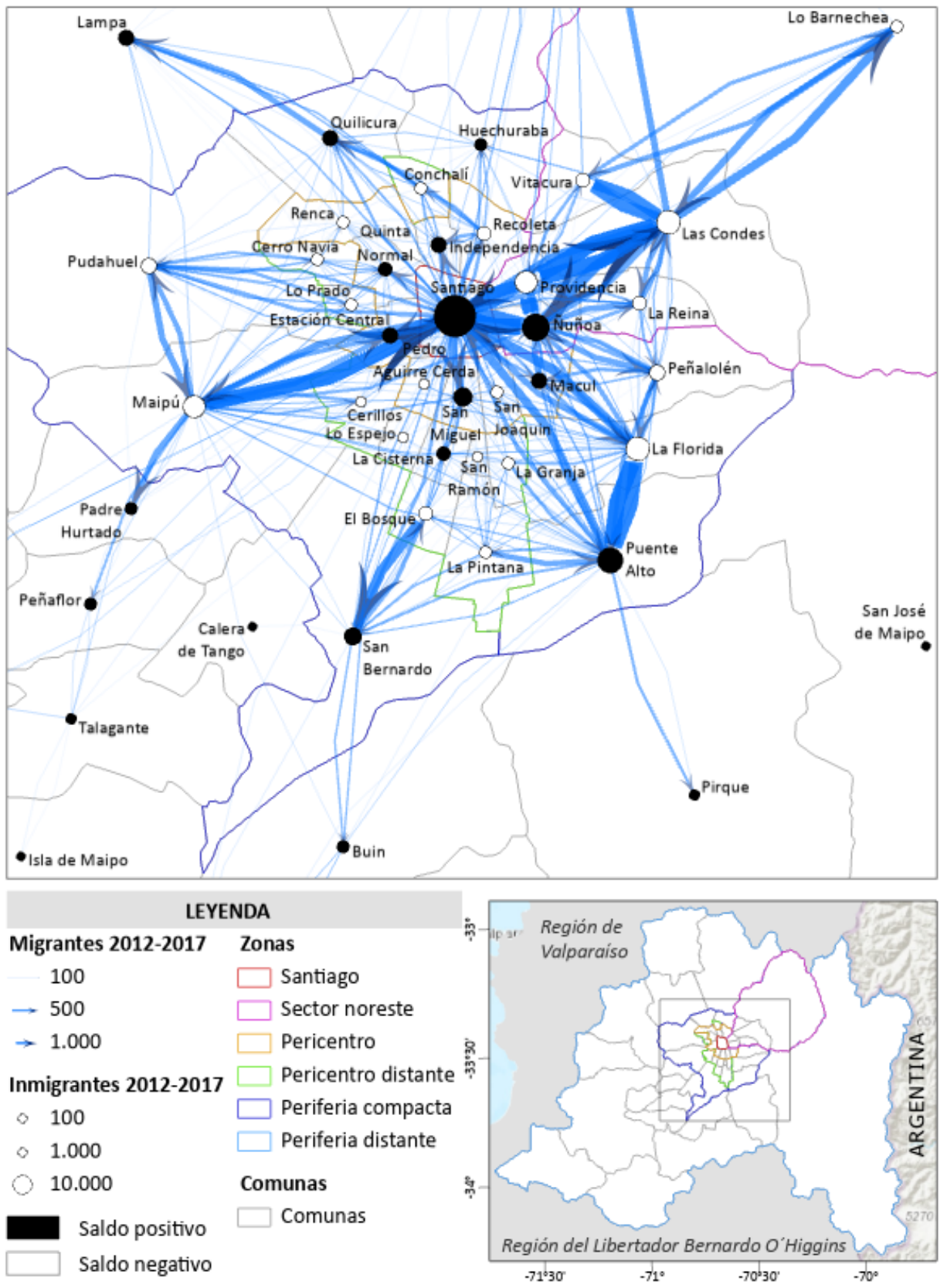

Información cartográfica

$\begin{array}{lllll}0 & 4 & 7 & 10 & \text { Referencia espacial }\end{array}$

Nombre: WGS 1984 UTM Zone 19S

Fuente: INE: Censo de población y vivienda 2017. RedAtam Webserver. Elaboración propia 
Los tipos de movimiento, las ocupaciones de los jefes de hogar y la distancia de los desplazamientos son aspectos que presentan también patrones significativos (Cuadro $\mathrm{N}^{\circ} 2$ ).

\section{Cuadro $\mathrm{N}^{\circ} 2$}

Movilidad residencial de los jefes de hogar por tipos de movimiento y ocupación en la RMS. 20122017

\begin{tabular}{|c|c|c|c|c|}
\hline \multirow[b]{2}{*}{ Tipo de movimiento } & \multicolumn{2}{|c|}{ Magnitud del flujo } & \multicolumn{2}{|c|}{ Ocupación de los jefes de hogar $\left(^{*}\right)$} \\
\hline & Absoluta & $\%$ del total & $\begin{array}{c}\text { Profesionales } \\
\text { (rama M) }\end{array}$ & \begin{tabular}{|c} 
No diferenciados \\
$($ rama $T)$
\end{tabular} \\
\hline Centralización & 47.464 & 14,9 & 2.324 & 1.365 \\
\hline Recentralización & 61.870 & 19,4 & 2.778 & 1.787 \\
\hline Suburbanización & 60.866 & 19,1 & 2.612 & 78 \\
\hline Lateral suburbano & 51.910 & 16,3 & 2.005 & 952 \\
\hline Al sector noreste & 26.166 & 8,2 & 2.865 & 958 \\
\hline Del sector noreste & 33.296 & 10,4 & 2.842 & 253 \\
\hline $\begin{array}{l}\text { Relocalización en el } \\
\text { sector noreste }\end{array}$ & 37.535 & 11,7 & 4.985 & 147 \\
\hline Total & 319.107 & 100,0 & 20.411 & 6.243 \\
\hline A Santiago & 33.798 & & 2.261 & 632 \\
\hline De Santiago & 30.616 & & 2.965 & 51 \\
\hline
\end{tabular}

$\left({ }^{*}\right)$ : Chi cuadrado: $\mathrm{P}<0,001$

Fuente: INE: Censo de población y vivienda 2017. RedAtam Webserver. Elaboración propia

En los mapas de las figuras $3 \mathrm{~A}$ y $3 \mathrm{~B}$ se representan los diferentes tipos de movimientos cuyo flujo es mayor de 25 jefes de hogar. Santiago es la comuna más diversificada por cuanto en ella fijan su residencia habitantes de todas las comunas de la RMS, y de aquí salen residentes al resto de las comunas; también es la comuna receptora más importante de los movimientos de centralización. Los flujos de recentralización son de reducida magnitud, pero tan numerosos que los sitúan en primer lugar por volumen absoluto, configuran una densa red, a modo de tela de araña, que expresa el dinamismo del mercado residencial de este sector. Los movimientos suburbanos son cuantiosos, parten principalmente de Santiago y, aunque muestran algunas concentraciones, se dispersan en todas las direcciones. Los flujos laterales suburbanos son especialmente intensos entre algunas comunas vecinas. Los flujos del sector noreste se caracterizan por sus fuertes intercambios internos y con Santiago. 
Figura $\mathrm{N}^{\circ} 3 \mathrm{~A}$

Flujos migratorios a Santiago, de Santiago, de centralización y recentralización. 20122017

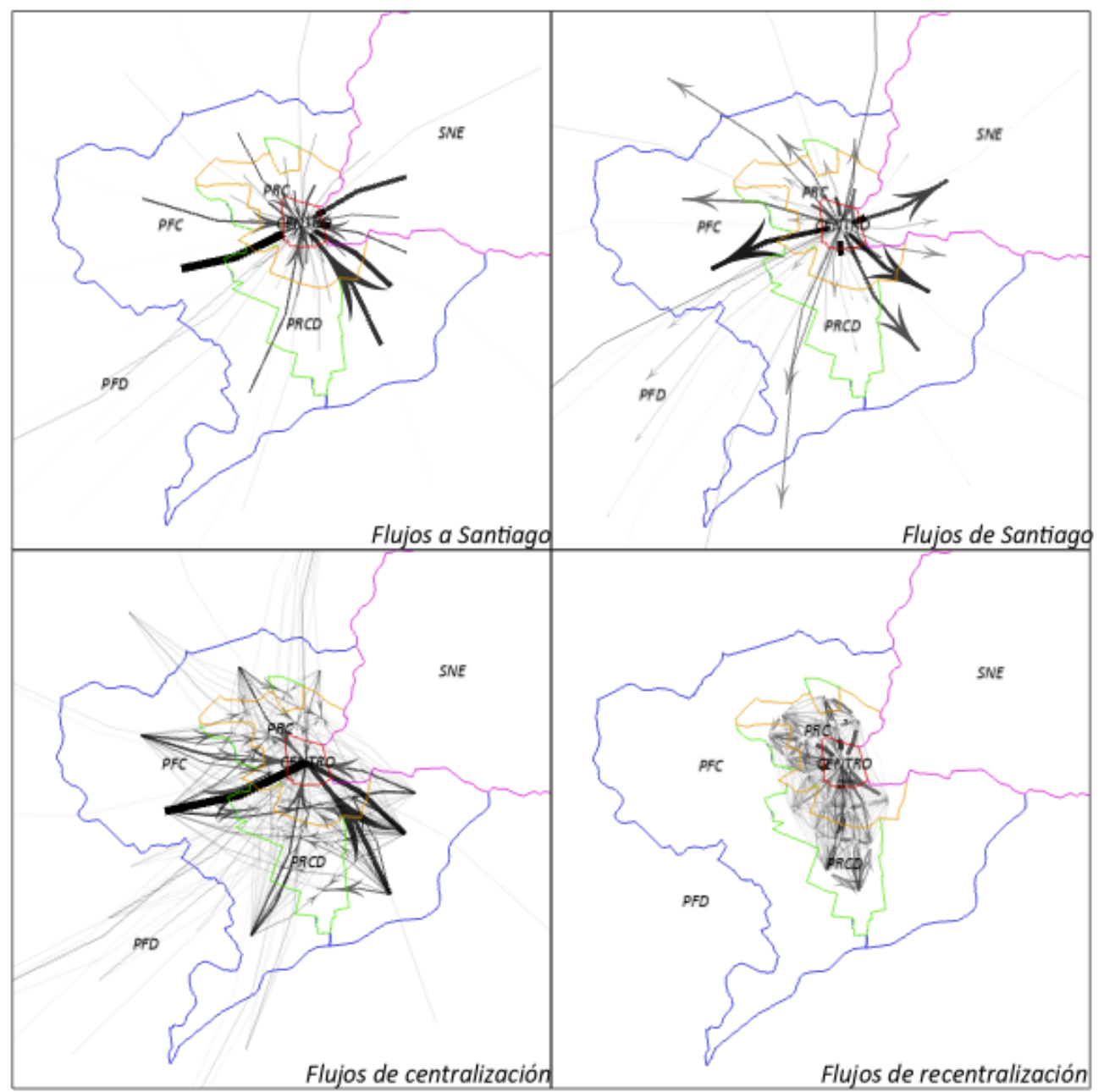

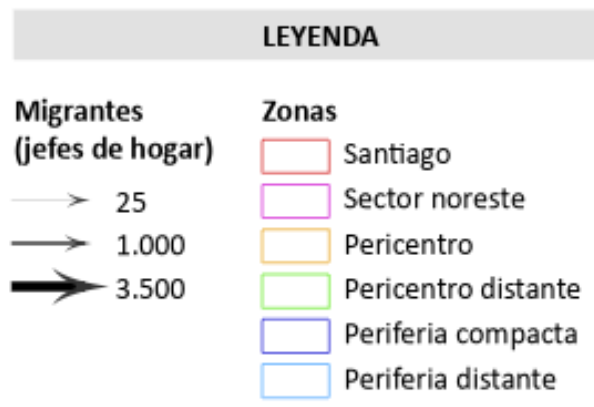

Información cartográfica
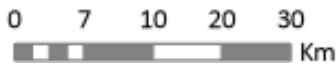

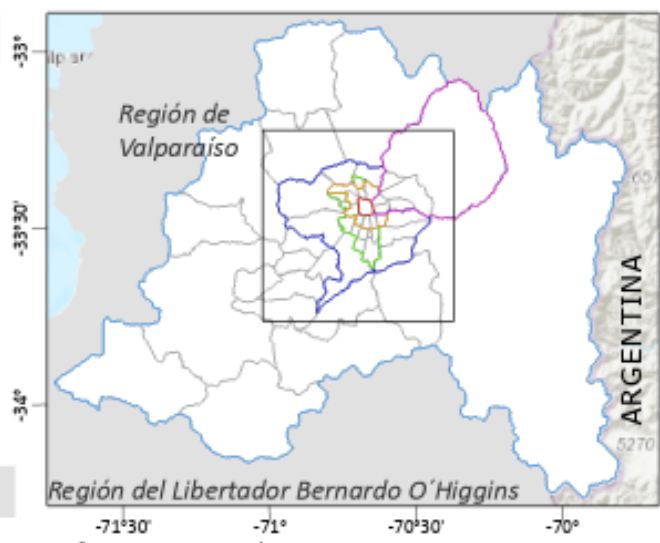

Referencia espacial Nombre: WGS 1984 UTM Zone 19S

Fuente: INE: Censo de población y vivienda 2017. RedAtam Webserver. Elaboración propia. 
Figura $\mathrm{N}^{\circ} 3 \mathrm{~B}$

Flujos migratorios de suburbanización, laterales suburbanos y del sector noreste. 20122017

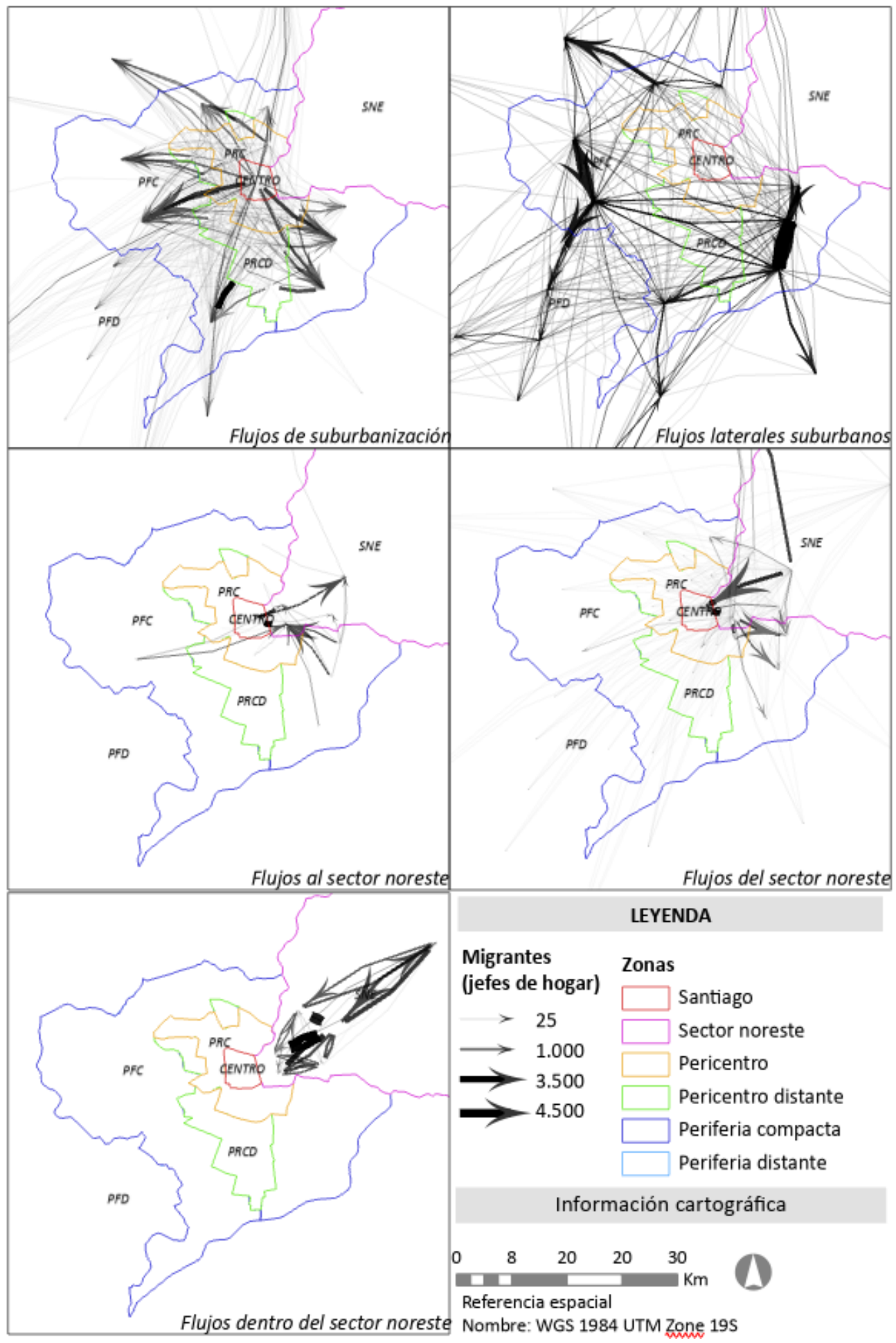

Fuente: INE: Censo de población y vivienda 2017. RedAtam Webserver. Elaboración propia. 
Figura No 4

Movimientos residenciales de elitización y ascenso socioeconómico en la RMS (más de 250 residentes). 20122017

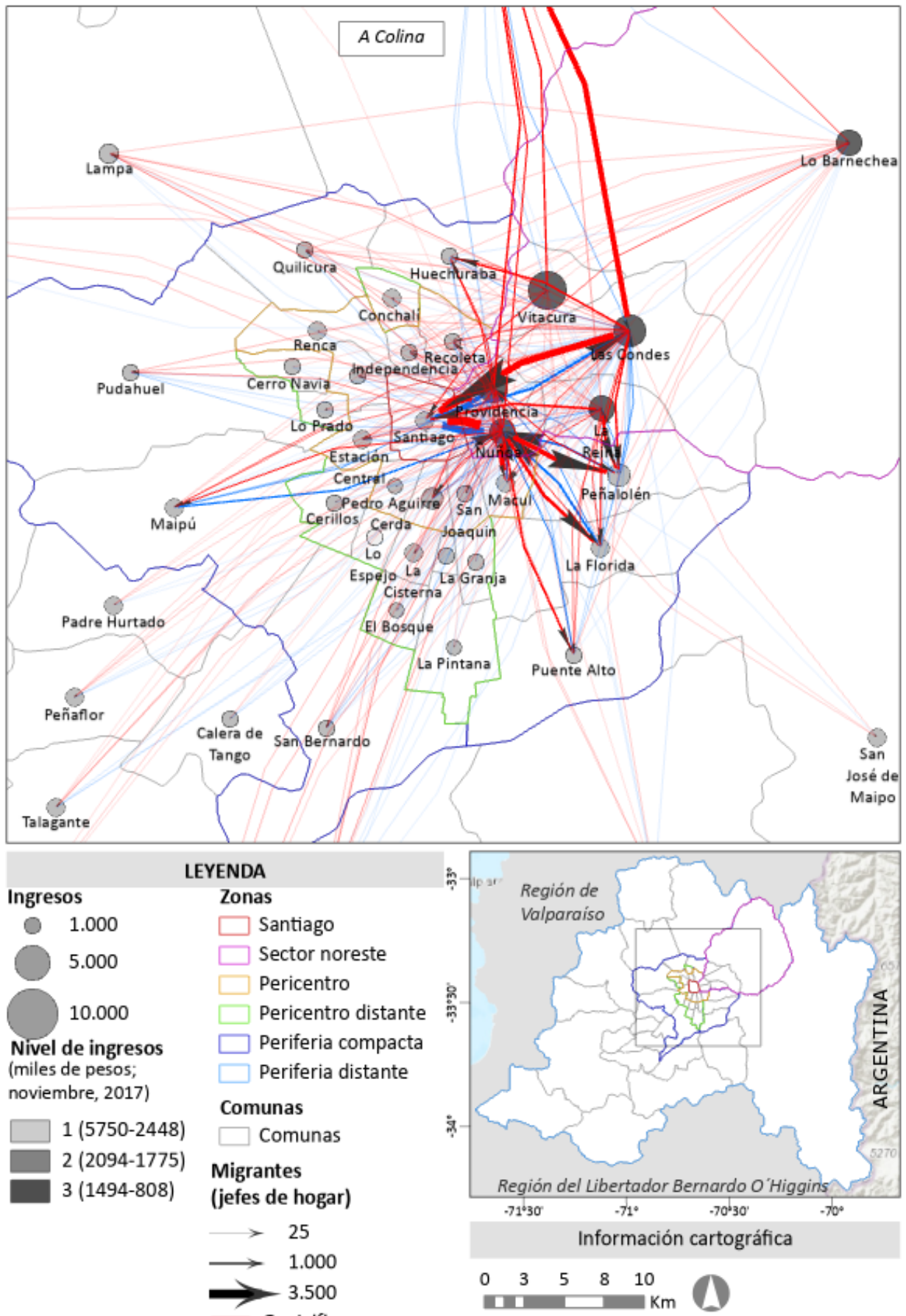

Gentrifiers

Escalators

Referencia espacial Nombre: WGS 1984 UTM Zone $19 S$

Fuente: INE: Censo de población y vivienda 2017. RedAtam Webserver. Elaboración propia. 
El grado de movilidad intercomunal de los jefes de hogar varía con la ocupación, pues del total de jefes de hogar ocupados en la rama $M$ (profesionales), el $24,1 \%$ han cambiado de comuna de residencia entre 20122017, frente al 10,8\% de los jefes de hogar ocupados en la rama $T$ (no diferenciados). Desde el punto de vista de los tipos de movimiento, las ocupaciones profesionales muestran una preferencia destacada por los movimientos de suburbanización y los internos en el sector noreste (Cuadro $N^{\circ} 2$ ).

A partir de los datos de «ingreso total del hogar» se ha construido la tipología de la figura № 4. En la misma se aprecia como los cambios residenciales de las comunas del noreste contribuyen a la diversificación selectiva de la periferia norte y sur, al mismo tiempo que otros residentes abandonan las comunas de menores ingresos, lo que refleja, probablemente, un ascenso socioeconómico de los migrantes.

Finalmente, examinamos la distribución de las conexiones bilaterales en función de su longitud y volumen de cambios de residencia.

En primer lugar, se constata que la distribución de los cambios residenciales según la distancia de sus desplazamientos se aparta de la distribución empírica de todas las distancias posibles entre comunas (distribución nula, HO). Como se observa en la figura No 5, la mayor parte de los cambios de residencia se produce a distancias relativamente cortas del domicilio anterior: la frecuencia máxima corresponde al tramo de 5-10 km; hasta $15 \mathrm{~km}$ se comprende el $66 \%$ de todos los cambios residenciales. La proporción de jefes de hogar que traslada su residencia a menos de $20 \mathrm{~km}$ está por encima de la proporción de la distribución nula, mientras que los que se hacen a mayor distancia están por debajo. Este perfil es similar en todos los tipos de movimiento en relación con sus respectivas distribuciones nulas (HO). En conjunto, la distribución de los cambios residenciales según la distancia se ajusta muy bien a una ley exponencial negativa (exponente: -0,42). Estos resultados son consistentes con los obtenidos en otros estudios (Potrykowska 1993; Trépanier 1978).

Figura No 5

Distribución de los flujos residenciales de los jefes de hogar según su longitud en las comunas de la RMS y distribución empírica de todas las distancias posibles entre los centroides de comuna (HO)

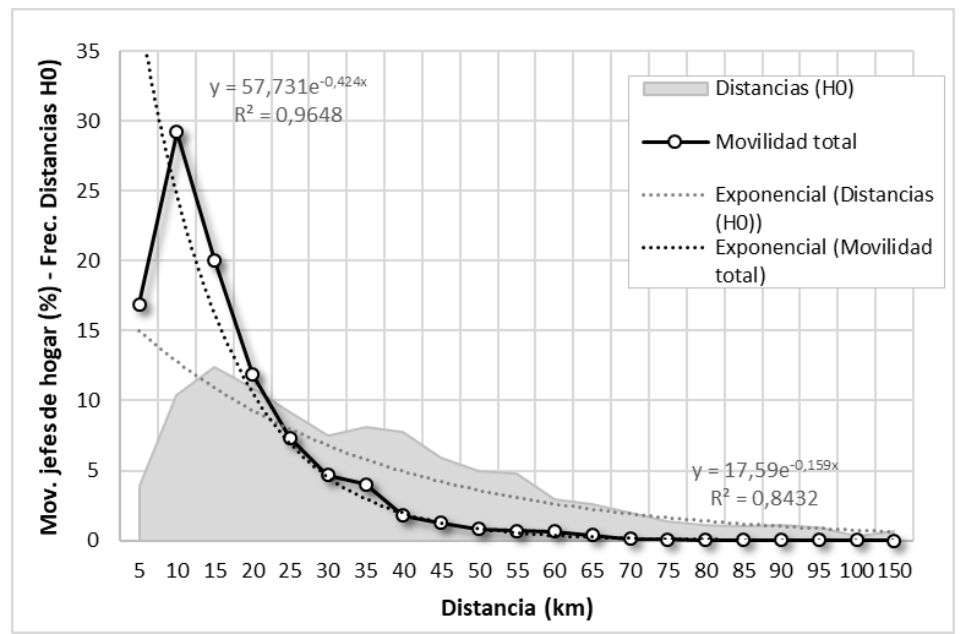

Fuente: INE: Censo de población y vivienda 2017. RedAtam Webserver. Elaboración propia. 


\section{Estructura espacial: modelo sectorial ¿en vías de disolución?}

En la imagen de la figura $N^{\circ} 6$ se puede observar que la red de cambios de residencia forma una estructura única, relativamente compleja, organizada en grupos y subgrupos de comunas con intensos intercambios migratorios, conectados y jerarquizados. La posición de cada comuna en el plano de la figura, es decir su distancia y orientación, expresa su grado de atracciónrepulsión con las demás, estimado por la intensidad de sus conexiones: la atracción es más intensa si los flujos mutuos de migrantes son mayores y menos intensa si un flujo es elevado y otro no o ambos, salidas y entradas, son débiles. En el primer caso, las comunas están próximas en la imagen y más alejadas en el segundo (compárese esta figura con el mapa de la figura 2).

Los rasgos estructurales de esta red se pueden resumir del modo siguiente: contiene grandes nodos que son los principales hub del sistema, pues reciben flujos considerables y definen los destinos de la movilidad residencial (Santiago, Ñuñoa, Puente Alto y otros); apenas existen agrupamientos de pequeño tamaño y ninguno de ellos está aislado; el nivel principal está formado por tres grandes agrupamientos - que conforman las tres ramas de la imagen-, cuyo punto de

Figura $N^{\circ} 6$

Estructura de los flujos migratorios residenciales en las comunas de la RMS (flujos mayores de 15 jefes de hogar). 20122017.

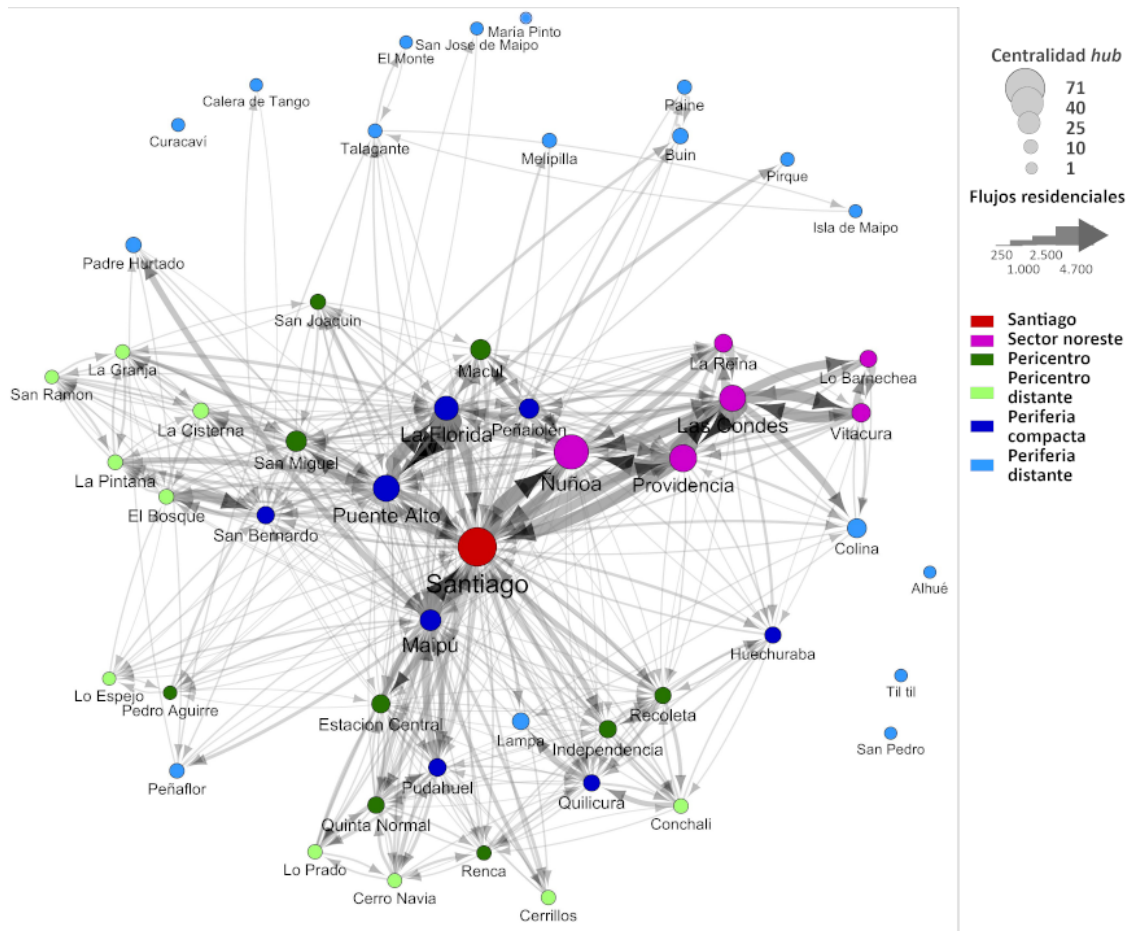

Fuente: INE: Censo de población y vivienda 2017. RedAtam Webserver. Elaboración propia. 
articulación fundamental es Santiago, que actúa como hub principal. Cada uno de estos tres grandes clústeres tiene una composición diferente en cuanto al sector de localización de las comunas que los integran. Uno de ellos está constituido, casi exclusivamente, por comunas del sector noreste de la RMS, con Ñuñoa y Providencia como importantes comunas distribuidoras; en los otros dos clústeres, vertebrados por Maipú y Puente Alto y La Florida, se mezclan comunas del pericentro y de las periferias; también se puede observar en la imagen que casi todas las comunas periféricas están más fuertemente vinculadas a Santiago que las del pericentro. Otra característica destacable en la red es la unión, a modo de puente, que se establece entre dos agrupamientos (el del eje derecho y el del eje izquierdo de la imagen) a través de varias comunas (Peñalolén, La Florida, Macul).

El procedimiento analítico aplicado para identificar los agrupamientos funcionales ha producido un valor máximo de modularidad $\mathrm{Q}=0,26$ con 4 grupos, todos ellos espacialmente continuos. Este valor moderado indica que en el conjunto de la red está bastante cohesionado. Los valores del índice El muestran, en efecto, que las relaciones internas entre las comunas de los módulos son idénticas a las externas, con diferencias leves. El clúster más grande en población y movilidad residencial es el «sureste» y el más pequeño el «noroeste» (cuadro No 3; figura 7). Esta configuración espacial se ha simplificado y ha incrementado su integración respecto de la del período 19972002, que con el mismo valor $Q=0,26$, presentaba 5 módulos, uno de ellos, la periferia, espacialmente discontinuo.

\section{Cuadro $\mathrm{N}^{\circ} 3$}

Datos generales de los módulos de la movilidad residencial de los jefes de hogar en las comunas de la RMS 2017

\begin{tabular}{|c|c|c|c|c|c|c|c|c|c|}
\hline \multirow{2}{*}{ Grupo } & \multirow{2}{*}{ Comunas } & \multirow{2}{*}{$\begin{array}{c}\text { Índice } \\
E_{w} I_{w}\end{array}$} & \multirow{2}{*}{$\begin{array}{c}\text { Población } \\
\text { Total (Mi- } \\
\text { les) }\end{array}$} & \multicolumn{2}{|c|}{ Movilidad (miles) } & \multirow{2}{*}{$\begin{array}{c}\text { Comunas } \\
(\%)\end{array}$} & \multirow{2}{*}{$\begin{array}{c}\text { Población } \\
(\%)\end{array}$} & \multicolumn{2}{|c|}{ Movilidad (\%) } \\
\hline & & & & Interna & Externa & & & Interna & Externa \\
\hline 1 (NE) & 8 & $-0,14$ & 1480 & 59 & 45 & 15,4 & 20.8 & 34,1 & 30,9 \\
\hline $2(\mathrm{SO})$ & 18 & 0,05 & 1830 & 32 & 35 & 34,6 & 25.7 & 18,3 & 23,9 \\
\hline $3(\mathrm{NO})$ & 8 & 0,11 & 963 & 17 & 21 & 15,4 & 13.5 & 9,7 & 14,4 \\
\hline 4 (SE) & 18 & $-0,19$ & 2840 & 66 & 45 & 34,6 & 39.9 & 37,9 & 30,8 \\
\hline Total & 52 & & 7113 & 174 & 146 & 100,0 & 100,0 & 100,0 & 100,0 \\
\hline
\end{tabular}

Fuente: INE: Censo de población y vivienda 2017. RedAtam Webserver. Elaboración propia. 
Figura No 7

Módulos de la movilidad residencial de los jefes de hogar en las comunas de la RMS. 20122017 (arriba)

Módulos: 2012-2017

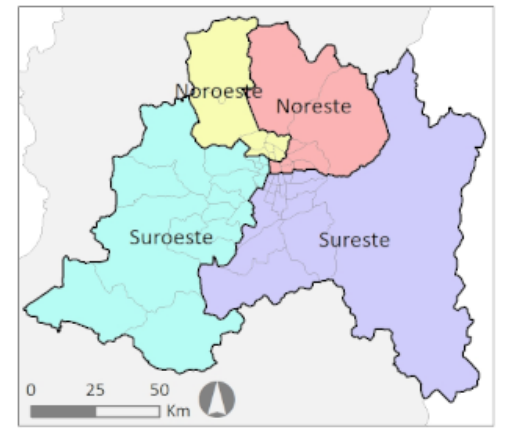

Módulos 1997-2002

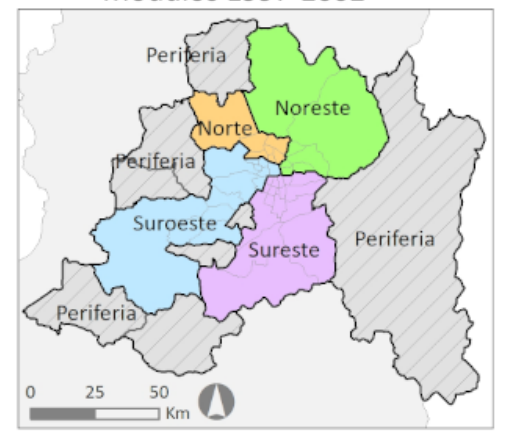

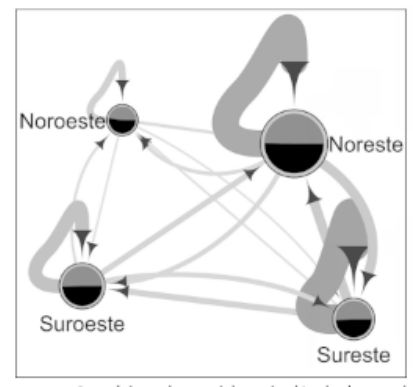

Cambios de residencia (J. de hogar) 200.000
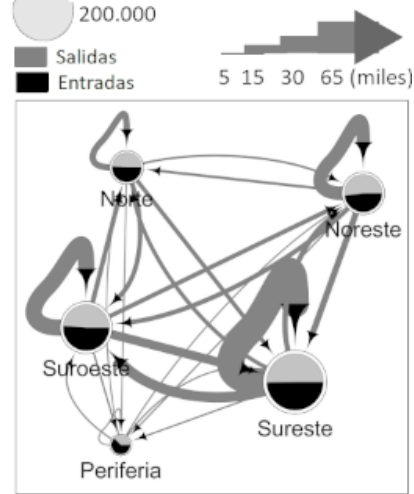

Fuente: INE: Censo de población y vivienda 2017. RedAtam Webserver. Elaboración propia.

\section{Movilidad residencial y dinámica metropolitana: procesos consolidados y emergentes}

Los resultados obtenidos se pueden resumir por cuatro características interrelacionadas de la movilidad residencial de los jefes de hogar en la RMS: a) la intensidad de la movilidad residencial es muy parecida en todas las comunas, y algo más elevadas las salidas en las comunas más grandes; b) el origendestino de la movilidad forma una red que comprende todo el territorio metropolitano y cuya integración es mayor con el paso del tiempo, pero que presenta varios nodos vertebradores y densidades y estructuras espacialmente diferenciadas; $c$ ) predominan los cambios de residencia a corta distancia y $\mathrm{d}$ ) el balance migratorio favorece a la periferia y a varias comunas del centro y es producido por complejos movimientos de suburbanización, centralización y de reorganización en la periferia y en el centro. En suma: una estructura espacial fruto de la combinación de proximidad y conexión aespacial que revela la relación entre la movilidad residencial y los procesos socioespaciales de reconfiguración y expansión de la RMS.

La mayor parte de los cambios de residencia se producen a corta distancia (a los $10 \mathrm{~km}$ del domicilio actual decae la frecuencia de la movilidad; figura $\mathrm{n}^{\circ} 5$ ), lo que confirma una de las ten- 
dencias más constantes de la movilidad humana (Potrikoska, 1993; Ravenstein, 1885; Trépanier, 1978), que tiene importantes implicaciones sociales y espaciales. Por una parte, la elección de nueva residencia en las cercanías del domicilio habitual expresa la fuerte asociación entre el comportamiento residencial y la valoración del capital social (Putnam, 1995) y de la percepción del espacio cotidiano. La permanencia en el mismo barrio o vecindad se valora como una cualidad fundamental de la nueva residencia, es decir, si todas las demás condiciones se mantienen iguales, se prefiere una residencia nueva en el entorno social y urbano ya conocido. Incluso, se pueden intercambiar algunos inputs (incremento de precio, reducción de la superficie) para adquirir una vivienda cercana a la actual; esta vinculación es especialmente fuerte en la clase mediaalta (Fuentes \& MacClure, 2019; Álvarez, 2010). Por otra, se trata de cambios residenciales que, muy probablemente, no van acompañados de un salto de nivel social o económico del sujeto que cambia de residencia, como en los casos de La FloridaPuente Alto, El BosqueSan Bernardo, PudahuelMaipú, MaipúPadre Hurtado o ConchalíQuilicura, excepto en las áreas de contacto de comunas de bajo y medio nivel de ingresos con las de elevado nivel de ingresos (VitacuraHuechuraba). En conjunto, distancia social y distancia espacial son pequeñas en esta categoría de movilidad residencial, salvo en el caso citado de fuertes contrastes en los niveles de renta entre comunas vecinas.

Por otra parte, la movilidad residencial de larga distancia se corresponde con salidas de residentes de comunas del centro hacia las periferias, próxima (o de la ciudad compacta) y lejana, de la periferia al centro y de algunas comunas periféricas del noreste hacia otras comunas también de la periferia. Muy probablemente, estos cambios espaciales van acompañados de otros cambios sociales o están motivados por condicionantes de mayor peso que la proximidad, como variaciones en la composición de la familia, en la localización del lugar de trabajo, búsqueda de mayor seguridad, etc. En estos casos, la distancia social en relación con la situación de partida y la distancia a la nueva residencia pueden ser considerables (MaipúSantiago; Puente AltoSantiago; La FloridaSantiago). Estos movimientos son explicables por los procesos de «evolución natural» (suburbanización) y «fligh from blight» (fiscalsocial approaches) (Mieszkowski \& Mills 1993, pp. 137-138; Bayoh et al., 2006; Morris et al., 2018; Andrews \& Caldera, 2011).

Las distancias de los cambios de residencia se combinan con su dirección origendestino, lo que da lugar a una tipología variada de flujos residenciales, muy relacionados con la segmentación social y espacial del mercado de la vivienda (Cox \& Hurtubia, 2016; Hidalgo et al., 2007). En este contexto, cobran sentido el considerable volumen de los movimientos residenciales de suburbanización, así como los cambios de residencia dentro y entre las zonas de la periferia. En el primer caso, los flujos de suburbanización más cuantioso parten de Santiago (es la tercera comuna más poblada) seguidos, como se ha dicho, de flujos de áreas vecinas. Los movimientos residenciales entre las comunas de ambas periferias son tan importantes que constituyen la fuente principal de movilidad residencial de estos espacios: el origen primordial de residentes de la periferia distante está en las comunas de la periferia compacta y en las propias comunas de la periferia distante; en la periferia compacta, los cambios residenciales más cuantiosos se generan entre sus propia comunas. Buena parte de estos cambios son debidos, seguramente, a la búsqueda de viviendas más acordes con las expectativas residenciales, pero se prefiere permanecer en la misma zona de la residencia actual (Pirque y San José de Maipo de residentes de Puente Alto; Buin de residentes de Paine). Sin embargo, otra parte de estos cambios se produce a larga distancia y están vinculados, muy probablemente, con las aspiraciones a viviendas en zonas muy específicas y exclusivas, con 
tipologías residenciales de condominios. Ambos tipos de movimiento residencial, de suburbanización y laterales, están asociados, con gran probabilidad, al mercado de vivienda nueva.

Los movimientos residenciales de centralización se relacionan con el hecho de que Santiago es el principal núcleo de empleo de la RMS, y la mayor concentración de ocupaciones de sector servicios (Rodríguez 2012; Escolano-Utrilla et al., 2015). Estos movimientos alcanzan todo el espacio de la RMS, aunque son más intensos en el sector noreste y con la comuna de Maipú. Los análisis han revelado también el gran dinamismo en cuanto a movilidad residencial de los pericentros, especialmente el inmediato a Santiago, lo que es un indicador de la formación de un potente mercado de viviendas, nuevas y de alquiler, impulsado por los procesos de renovación urbana (y gentrificación) llevados a cabo en el centro y pericentro.

Las comunas del sector noreste presentan el grado más elevado de endogamia residencial, ya que la mayor parte de los cambios residenciales se producen entre las comunas del mismo sector (Las Condes, Lo Barnechea, Vitacura, La Reina). Los resultados obtenidos concuerdan con las conclusiones de Méndez \& Gayo (2019) y producen la acumulación de población de mayores ingresos en este sector (Hidalgo el al. 2007, p.122).

Los cambios residenciales descritos inciden en el aumento (o descenso) de la segregación y de la diversificación socioespacial, así como en la estructura demográfica de las áreas metropolitanas (Susino, 2010). Los resultados obtenidos están en línea con la intuición de Rodríguez Vignoli y Rowe $(2018$, p. 12) acerca de la diversificación social de la periferia de Área Metropolitana de Santiago (AMS), donde se han creado nuevas urbanizaciones cerradas de residentes de elevados ingresos y niveles educativos: «...Aunque no hay evidencia sólida sistematizada, se suele suponer que una fracción importante de los residentes de estas nuevas urbanizaciones de lujo correspondería a familias acomodadas que residían en el hábitat histórico de la clase alta». Las diferencias espaciales y de intensidad de los cambios de residencia de los jefes de hogar según su ocupación (profesionales y no diferenciados) ponen de manifiesto la existencia de subredes de movilidad particulares asociadas, probablemente, a mercados específicos de vivienda (condominios de alto nivel, viviendas sociales, etc.) y a determinados grupos sociales.

La relocalización residencial de jefes de hogar procedentes de comunas de altos ingresos afecta, casi exclusivamente, a la mitad oriental de la ciudad, lo que concuerda con las investigaciones de Rodríguez Vignoli y Rowe (2017), que han demostrado la influencia dual de la movilidad residencial en la modificación de la sociogeografía del AMS: por un lado, han contribuido decisivamente a la diversificación socioeducativa de la periferia, en la que se han formado núcleos de residentes con elevada formación pero, por otro, han reducido la proporción de población de este nivel educativo en el pericentro. El balance de esta doble tendencia hace que persistan los elevados niveles de segregación residencial, aunque se observe cierta diversificación del espacio residencial (Sabatini et al., 2001).

Finalmente, los análisis han evidenciado que el conjunto de cambios de residencia adquiere una configuración sectorial, asimilable a un modelo de tipo hoytiano (Hoyt 1939) semejante a la detectada en otras ciudades (Sun \& Manson, 2016). El dominio de los movimientos radiales de las trayectorias espaciales de la movilidad, que alinean la residencia con el centro y la periferia de la RMS genera, a macroescala, cuatro grandes sectores. Esta estructura hace que cada sector 
tenga cierta heterogeneidad social y espacial, pues comprende espacios centrales, intermedios y periféricos, entre los que se producen la mitad de los cambios de residencia, centrífugos o centrípetos, cortos o largos; la otra mitad se produce con las restantes comunas de la RMS, es decir, buena parte de los procesos de diversificación social y funcional se producen en el interior de los sectores delimitados. El caso de la expansión de la periferia urbana, la ocupación residencial se puede asimilar a un modelo de crecimiento de ondas que se van alejando del centro, perturbado por otros movimientos de larga distancia.

\section{Conclusiones}

Los resultados obtenidos permiten afirmar que los movimientos residenciales dentro de la RMS son vectores fundamentales de la suburbanización de la población y del crecimiento de la periferia al mismo tiempo que se integran en la potente dinámica funcional y residencial del centro de la región. Y también han evidenciado que las tres cuartas partes de los cambios de residencia se producen en las cercanas de la residencia anterior.

Sin embargo, su capacidad como agentes de diversificación social es limitada y selectiva, ya que la mayor parte de los cambios residenciales se producen entre comunas vecinas o muy cercanas $y$, además, dentro de sectores espaciales que conectan el centro con la periferia. Buena parte de la movilidad residencial responde a un proceso de difusión espacial, filtrado por sectores.

En conjunto, el modelo de movilidad residencial a escala comunal reproduce, grosso modo, el modelo espacial, social y funcional de la RMS y también forma parte de la imagen espacial que de sí mismas, y de otros grupos sociales, tienen las clases medias. Es sorprendente la similitud del mapa de la estratificación socioespacial de las comunas de Santiago según las clases medias, elaborado por Fuentes y MacClure (2019: 7), y el de la figura 7.

El método utilizado ha permitido identificar la estructura funcional de red de la movilidad residencial de la RMS y delimitar las áreas espaciales a partir de los datos dinámicos de flujos residenciales. Los resultados han puesto de manifiesto relaciones y efectos que no se detectan con el uso de atributos estáticos. Una vez establecidos los patrones geográficos de la movilidad residencial agregada, se propone, como líneas lógicas de investigación futura, extender el estudio de la movilidad residencial a otras áreas metropolitanas de América Latina aplicando esta metodología y caracterizar, a diferentes escalas, los modelos migratorios de algunos grupos sociales, definidos por la ocupación, el nivel de cualificación, la edad y el tamaño de los hogares.

\section{Anexo}

Ámbitos espaciales de análisis y comunas que los integran

Centro (1): Santiago

Pericentro (9): Estación Central, Independencia, Macul, Pedro Aguirre Cerda, Quinta Normal, Recoleta, Renca, San Joaquín, San Miguel. 
Pericentro distante (10): Cerrillos, Cerro Navia, Conchalí, El Bosque, La Cisterna, La Granja, La Pintana, Lo Espejo, Lo Prado, San Ramón.

Periferia compacta (8): Huechuraba, La Florida, Maipú, Peñalolén, Pudahuel, Puente Alto, Quilicura, San Bernardo.

Periferia distante (18): Alhué, Buin, Calera de Tango, Colina, Curacaví, El Monte, Isla de Maipo, Lampa, María Pinto, Melipilla, Padre Hurtado, Paine, Peñaflor, San José de Maipo, San Pedro, Talagante, Tiltil.

Sector noreste (6): La Reina, Las Condes, Lo Barnechea, Ñuñoa, Providencia, Vitacura.

\section{Bibliografía}

ÁLVAREZ, A.M. Tan lejos, tan cerca. Elección residencial, de localización y modos de vida urbanos en sectores de clase media de la Región Metropolitana de Santiago, Chile, tesis Santiago de Chile: Pontificia Universidad Católica de Chile, 2010. Disponible en: http://www.theses.fr/2013PEST1043

ANDREWS, D. \& CALDERA, A. Residential Mobility and Public Policy in OECD Countries. OECD Journal: Economic Studies, 2011, vol. 2011/ 1, p. 122. doi: https://doi.org/10.1787/eco_studies-2011-5kgOvswqt240.

BÄING, A. \& WONG, C. Brownfield Residential Development: What Happens to the Most Deprived Neighbourhoods in England? Urban Studies. 2012, vol. 49, N014, p. 29893008.

BAYOH, I., IRWIN, E. G. \& HAAB, T. Determinants of Residential Location Choice: How Important Are Local Public Goods in Attracting Homeowners to Central City Locations? Journal of Regional Science, 2006, vol. 46, No1, p. 97120. doi: http://doi.wiley.com/10.1111/j.0022-4146.2006.00434.x

BIBLE, D. S. \& BROWN, L. A. Place utility, attribute tradeoff, and choice behavior in an intra-urban migration context. Socio-Economic Planning Sciences, 1981, vol. 15, No1, p. 3744. doi: https://doi. org/10.1016/0038-0121(81)90016-1

BONACICH, P. Factoring and weighting approaches to status scores and clique identification. The Journal of Mathematical Sociology, 1972, vol. 2, N01, p. 113120. doi: https://doi.org/10.1080/0022250X.1972.9989806

BORSDORF, A. Cómo modelar el desarrollo y la dinámica de la ciudad latinoamericana. EURE, 2003, vol. 29, No 86, p. 3749.

BORSDORF, A. \& HIDALGO, R. From polarization to fragmentation. Recent changes in Latin American urbanization. En: LINDER, P. V. \& VERKOREN, O. Decentralized development in Latin America: Experiences in local governance and local development. Dordrech, Heidelberg, Londres y Nueva York: Springer, 2010, p. 2334. 
BROWN, L A \& MOORE, E G. The Intra-Urban Migration Process: A perspective. Geografiska Annaler. Series B, Human Geography, 1970, vol. 52, p. 113.

BRUMMELL, A. C. A Model of Intraurban Mobility. Economic Geography, 1979, vol. 55, № 4, p. 338352. doi: $10.2307 / 143165$

CARLEY, K. M, ORA-NET SCENES. Center for Computational Analysis of Social and Organizational Systems (CASOS), Institute for Software Research International (ISRI), School of Computer Science, Carnegie Mellon University, 2017.

CLARK, W. A. V. \& DIELEMAN, F. M. Households and housing: choice and outcomes in the housing market. New Brunswick, N.J: Center for Urban Policy Research, 1996.

CLAUSET, A., NEWMAN, M. E. J. \& MOORE, C. Finding community structure in very large networks. Physical Review E, 2004, vol. 70, №. 6. Disponible en Internet: https://link.aps.org/doi/10.1103/ PhysRevE.70.066111

COULTER, R., HAM, M. van \& FINDLAY, A. M. Re-thinking residential mobility: Linking lives through time and space. Progress in Human Geography, 2016, vol. 40, No 3, p. 352374. Doi: https://doi. org/10.1177/0309132515575417

COX, T. \& HURTUBIA, R. Vectores de expansión urbana y su interacción con los patrones socioeconómicos existentes en la ciudad de Santiago. EURE Revista Latinoamericana de Estudios Urbano Regionales, 2016, vol. 42, No. 127, p. 185207.

DE MATTOS, C A., DUCCI, M E, RODRÍGUEZ, A \& YÁÑEZ, G (eds.), Santiago en la globalización: ¿una nueva ciudad? Santiago: Ediciones SUR; EURE Libros, 2004

DE MATTOS, C.A., \& HIDALGO, R. (eds.). Santiago de Chile: movilidad espacial y reconfiguración metropolitana. Santiago, Chile: Inst. de Estudios Urbanos y Territoriales, Pontificia Univ. Católica de Chile, Colección EURE-Libros, 8, 2007.

DI VIRGILIO, M. M. La movilidad residencial: una preocupación sociológica. Territorios, 2011, vol. 25, p. 173190 .

ESCOLANO-UTRILLA, S., ORTIZ-VÉLIZ, J. \& MORENO-MORA, R. La estructura de los núcleos de empleo en la región metropolitana de Santiago (Chile): centralización funcional y espacial. Boletín de la Asociación de Geógrafos Españoles, 2015, vol. 69, p. 263290.

FUENTES, Luis \& MAC-CLURE, Oscar, 2019. The middle classes \& the subjective representation of urban space in Santiago de Chile. Urban Studies, 2019, Doi: https://doi.org/10.1177/0042098019881350

HERRERA-PONCE, M. S. Movilidad residencial en la Región Metropolitana de Santiago: ¿hacia un mayor asentamiento poblacional? comparación con los datos de los censos 1992 y 2002. En: DE 
MATTOS, C. HIDALGO, R. Santiago de Chile. Movilidad espacial y reconfiguración metropolitana. Santiago de Chile: Instituto de Geografía, Pontificia Universidad Católica de Chile, 2007, p. 6988.

HIDALGO, R. \& BORSDORF, A. \& SÁNCHEZ, R. La expansión residencial amurallada en la reconfiguración metropolitana de Santiago de Chile. En: DE MATTOS, C.A., \& HIDALGO, R. (eds.). Santiago de Chile: movilidad espacial y reconfiguración metropolitana. Santiago, Chile: Inst. de Estudios Urbanos y Territoriales, Pontificia Universidad Católica de Chile, colección EURE-Libros, 8, 2007, p. 117136.

HOYT, H. The Structure AND Growth of Residential Neighborhoods in American Cities. Washintong, DC: Federal Housing Administration, 1993.

INE. Censo de población y vivienda. REDATAM Procesamiento y diseminación. Disponible en Internet: https://redatam-ine.ine.cl/redbin/RpWebEngine.exe/Portal?BASE=CENSO_2017\&lang=esp

INE. Cuestionario Oficial Censo 2017. Disponible en Internet: http://www.censo2017.cl/cuestionario-censal_2017

KING, R. Geography and Migration Studies: Retrospect and Prospect. Population, Space and Place, 2012, vol. 18, No. 2, p. 134153. Doi: https://doi.org/10.1002/psp.685.

KLEINBERG, J. M. Decision algorithms for unsplittable flow and the half-disjoint paths problem. In: 30th ACM Symposium on Theory of Computing, ACM Press, 1998, p. 530539. Disponible en Internet: http://portal.acm.org/citation.cfm?doid=276698.276867

MALLIAROS, F. D. \& VAZIRGIANNIS, M. Clustering and community detection in directed networks: A survey. Physics Reports. December 2013, vol. 533, No. 4, p. 95142. Doi: https://doi.org/10.1016/j. physrep.2013.08.002.

MÉNDEZ, M. L. \& GAYO, M. Upper middle class social reproduction: wealth, schooling, and residential choice in Chile. New York, NY: Springer, 2018.

MÉNDEZ, M. L. \& GAYO, M. Social Mobility over Time and in Space: Ascending Residential \& Social Trajectories. En: MÉNDEZ, M. L. \& GAYO, M. Upper Middle Class Social Reproduction. Cham: Palgrave Macmillan, 2019, p. 2954. Disponible en Internet: https://doi.org/10.1007/978-3-319-89695-3_2

MIESZKOWSKI, P. \& MILLS, E. S. The Causes of Metropolitan Suburbanization. the Journal of EConomic Perspectives, 1993, vol. 7, No. 3, p. 135147.

MINISTERIO DE DESARROLLO SOCIAL Y FAMILIA. Observatorio Social. Resultados Encuesta CASEN 2015. Disponible en Internet: http://observatorio.ministeriodesarrollosocial.gob.cl

MORRIS, T., MANLEY, D. \& SABEL, C. E. Residential mobility: Towards progress in mobility health research. Progress in Human Geography, 2018, vol. 42, No 1, p. 112133. Doi: https://doi. org/10.1177/0309132516649454. 
NEWMAN, M. E. J. Modularity and community structure in networks. Proceedings of the National Academy of Sciences, 2006, vol. 103, No. 23, p. 85778582. Doi: https://doi.org/10.1073/ pnas.0601602103

ORTIZVÉLIZ, J. \& ESCOLANOUTRILLA, S. Movilidad residencial del sector de renta alta del Gran Santiago (Chile): hacia el aumento de la complejidad de los patrones socioespaciales de segregación, EURE, 2013, 39(118), p. 7796.

POTRYKOWSKA, A. Intra-urban migration in the Warsaw urban region. Geographia Polonica, 1993, vol. 61, p. 281292.

PUJADAS, I. PRATS, P. \& COLL, M. Elección residencial y nuevas formas urbanas: el caso de la Región Metropolitana de Barcelona. En: ARTIUES, A.A., BAUZÁ, A., BLÁZQUEZ, M., GONZÁLEZ, J.M., MURRAY, I. \& RULLÁN, O. Los procesos urbanos postfordistas. Palma de Mallorca: Universitat de les Illes Balears y AGE, 2007, p. 215234.

PUTNAM, R.D., 1995. Bowling Alone: America's Declining Social Capital. Journal of Democracy, 1995, vol. 6, No 1, p. 6578. Doi: 10.1353/jod.1995.0002.

RAULIN, A. Anthropologie urbaine. Paris: Armand Colin.

RAVENSTEIN, E. G. The laws of migration-I, Journal of The Statistical Society, 1885, vol. 48, p. 167227

RODRÍGUEZ, J. ¿Policentrismo o ampliación de la centralidad histórica en el área Metropolitana del Gran Santiago? Evidencia novedosa proveniente de la encuesta Casen 2009. EURE, 2012, 38(114), p. 7197.

RODRÍGUEZ, J. \& ROWE, F. ¿Contribuye la migración interna a reducir la segregación residencial? El caso de Santiago de Chile 1977-2002. Revista Latinoamericana de Población, 2017, vol. 21, p. 746.

RODRÍGUEZ, J. \& ROWE, F. Efectos cambiantes de la migración sobre el crecimiento, la estructura demográfica y la segregación residencial en ciudades grandes: el caso de Santiago, Chile, 19772017, Santiago (Chile): CEPAL, Población y Desarrollo, 2018. Disponible en Internet: https:// www.cepal.org/es/publicaciones/44367

SABATINI, F, CÁCERES, G \& CERDÁ, J. Segregación residencial en las principales ciudades chilenas: Tendencias de las tres últimas décadas y posibles cursos de acción. EURE, 2001, 27(82), p. 2142.

SHANNON, P., MARKIEL, A., BALIGA, NS, WANG, J.T., RAMAGE, D., AMIN, N., SCHWIKOWSKI, B. \& IDEKER, T. Cytoscape: a software environment for integrated models of biomelecular interaction networks. Genome Research, 2003, Vol. 13, no. 11, p. 24982504 . Doi: doi:10.1101/gr.1239303

SUN, S. \& MANSON, S. M. Intraurban Migration, Neighborhoods, and City Structure. Urban Geography. 2012, vol. 33, No 7, p. 10081029. Doi: https://doi.org/10.2747/0272-3638.33.7.1008 
SUSINO, J. La movilidad residencial diferencial en la reconfiguración metropolitana. En: FERIA, J.M. \& ALBERTOS, J.M: La ciudad metropolitana en España: procesos urbanos en los inicios del siglo XXI. Thompson Reuters-Civitas, 2010, p. 149174.

TORRADO, J.M. Flujos espaciales y dinámicas residenciales de centralización en las áreas metropolitanas españolas. Granada: Universidad de Granada, 2019. Disponible en Internet: http://hdl. handle.net/10481/59770

TRÉPANIER, C. La mobilité dans l'agglomération de Trois-Rivières. Cahiers de géographie du Québec. 1978, vol. 22, No 57, p. 377. Doi: https://doi.org/10.7202/021410ar.

VAN DER VLIST, A.J., CZAMANSKI, D. \& FOLMER, H. Immigration \& urban housing market dynamics: the case of Haifa. The Annals of Regional Science, 2011, vol. 47, № 3, p. 585598. Doi: https://doi. org/10.1007/s00168-010-0396-2

VILLANI, T. Psychogéographies urbaines corps, territoires et technologies. Paris: Eterotopia. 2014. 
